Draft Version November 7, 2018

Preprint typeset using $\mathrm{LAT}_{\mathrm{E}} \mathrm{X}$ style emulateapj v. 11/10/09

\title{
TAKING ADVANTAGE OF THE COS TIME-TAG CAPABILITY: OBSERVATIONS OF PULSATING HOT DQ WHITE DWARFS AND DISCOVERY OF A NEW ONE
}

\author{
P. Dufour ${ }^{1}$, S. Béland ${ }^{2}$, G. Fontaine ${ }^{1}$, P. Chayer $^{3}$, And P. Bergeron $^{1}$ \\ Draft version November 7, 2018
}

\begin{abstract}
We present an analysis of the ultraviolet light curves of five Hot DQ white dwarfs recently observed with the Cosmic Origins Spectrograph (COS) on board the Hubble Space Telescope (HST). These light curves were constructed by extracting the time-tag information from the FUV and NUV spectroscopic data. Single-color light curves were thus produced in $60 \mathrm{~s}$ time bins. The Fourier analysis of these data successfully recovers the main pulsation modes of the three stars previously known to be variable from ground-based observations. We also report the discovery of pulsations in another object, SDSS J1153+0056, making it only the fifth member of the new class of variable Hot DQ stars, and the first pulsating white dwarf to be discovered from space-based observations. The relatively high amplitudes of the modes observed in the FUV - 2 to 4 times that observed in the optical - as well as the high fraction of stars variable in our sample suggest that most, if not all, Hot DQ white dwarfs might be pulsating at some level when observed at high enough sensitivity. Our results also underline the vast potential of the time-tag capability of the HST/COS combination.
\end{abstract}

Subject headings: stars: oscillations — stars: individual (SDSS J1016+1513, SDSS J1153+0056, SDSS J1337-0026, SDSS J1426+5752, SDSS J2200-0741)

\section{ASTROPHYSICAL CONTEXT}

White dwarfs stars are traditionally found to have surface compositions made primarily of hydrogen or helium. However, a new family has recently been uncovered, the so-called Hot DQ white dwarfs, which have surface compositions dominated by carbon with little or no trace of hydrogen and helium (Dufour et al. 2007, 2008). These stars are exceedingly rare, and only 14 Hot DQ white dwarfs have been found among the sample of spectroscopically-identified white dwarfs which now comprises more than 12,000 objects (Dufour et al. 2010). It is believed that the Hot DQ's could be the progenies of stars similar to the unique hot PG 1159 star H1504+65, and could represent a new evolutionary sequence that follows the asymptotic giant branch (Dufour et al. 2007). It is also possible that they evolved from stars that were initially massive enough to ignite carbon and form oxygen/magnesium/neon cores with a carbon/oxygen envelope.

Given the range of effective temperature in which Hot DQ white dwarfs are found $(18,000-24,000 \mathrm{~K}$, according to Dufour et al. 2008), and proceeding in analogy with the presence of instability strips along the white dwarf cooling sequences (the ZZ Ceti strip centered around $12,000 \mathrm{~K}$ for the H-dominated atmosphere white dwarfs, and the V777 Her strip centered around $25,000 \mathrm{~K}$ for the He-dominated atmosphere white dwarfs), Montgomery et al. (2008) carried out a search for variability among them, which led to the discovery of the first pulsating Hot DQ white dwarf, SDSS

\footnotetext{
${ }^{1}$ Département de Physique, Université de Montréal, C.P. 6128, Succ. Centre-Ville, Montréal, QC H3C 3J7, Canada; dufourpa,fontaine,bergeron@astro.umontreal.ca

${ }^{2}$ Center for Astrophysics and Space Astronomy, 389 UCB, University of Colorado, Boulder, CO 80309, USA;sbeland@colorado.edu

${ }^{3}$ Space Telescope Science Institute, 3700 San Martin Drive, Baltimore, MD 21218, USA; chayer@stsci.edu
}

J1426+5752. The hypothesis that the luminosity variations seen in that star are caused by pulsational instabilities associated with low-order and low-degree gravitymode oscillations (as in the other known types of pulsating white dwarfs) is backed by the exploratory nonadiabatic calculations carried out by Fontaine, Brassard , \& Dufour (2008).

Since then, three new members have been added to this new class of variable white dwarf stars (Barlow et al. 2008; Dunlap et al. 2010), thus opening up the exciting possibility of probing the internal structure and testing the proposed formation scenarios of Hot DQ's through asteroseismological means.

In order to exploit fully the asteroseismological potential and better understand the origin and evolution of Hot DQ white dwarfs, accurate determinations of the atmospheric parameters and physical properties (effective temperature, surface gravity, mass, surface composition) are first necessary. Given the unusual atmospheric compositions in particular, it is necessary to improve upon the current estimates of these parameters. This requires better model atmospheres than currently available, incorporating the best physics and opacities available, notably of several carbon ions. This also requires better optical spectroscopic observations than those available through the Sloan Digital Sky Survey archives. Efforts on both of these fronts are currently being made and will be presented in due time.

In addition, at the effective temperatures where Hot DQ white dwarfs are found, most of the flux is emitted in the ultraviolet portion of the electromagnetic spectrum. According to model atmosphere calculations presented in Dufour et al. (2008), this region is particularly affected by the presence of numerous strong features of ionized carbon. These absorption features have a huge impact on the energy distribution and thermodynamic structure of the atmosphere because of flux redistribution. Hence, 
it is of utmost importance to be confident that the models correctly reproduce the UV portion of the spectrum.

In order to verify this explicitly, we requested and were awarded 33 orbits of HST time to observe Hot DQ white dwarfs with the Cosmic Origins Spectrograph (COS). Beyond the spectroscopic capability of COS, this instrument has also the capacity to observe in time-tag mode, making it an ideal instrument to measure time variabilities of astronomical objects in the ultraviolet. In the following, we thus focus on the temporal aspect of our observations (the spectroscopic analysis will be presented elsewhere), and we discuss the characteristics and properties of the light curves that we extracted from our recent HST/COS observations of five Hot DQ white dwarfs.

\section{OBSERVATIONS}

Our sample consists of five of the nine Hot DQ white dwarfs first analyzed in some detail by Dufour et al. (2008) on the basis of optical spectroscopy. These objects were chosen in order to cover a representative variety of observed Hot DQ properties, such as the presence of traces of helium, hydrogen, magnetic fields, and variability. At the time of the Cycle 17 call for proposals (March 2008), only SDSS J1426+5752 was known to exhibit pulsations and, as such, it was included in our sample despite its faintness $(\mathrm{g}=19.16)$. As a consequence, to reach the desired minimal signal-to-noise ratio of 20 in our spectroscopic observations, this object received the longest temporal coverage in our sample, a fortunate situation for time variability analysis (see below). Note also that, since the call for proposals, two of our scheduled targets have been found to show luminosity variations from ground-based observations, namely, SDSS J2200-0741 (Barlow et al. 2008) and SDSS J1337-0026 (Dunlap et al. 2010).

Because most of the carbon lines that dominate the spectra are very broad, low resolution observations with the FUV G140L and NUV G230L gratings were carried out. In order to cover as much as possible of the spectral energy distribution, three different nominal wavelength settings were used for the NUV observations, while one setting was used for the FUV region. This resulted in an almost complete coverage between $\sim 1200$ and $3000 \AA$ for each star. The light curves were extracted from the COS FUV and NUV observations taken in time-tag mode. In this mode, each photon has a time stamp for its detection with a resolution of $0.032 \mathrm{~s}$. Before processing the FUV data, thermal and geometric corrections were applied 4 (Béland \& Penton 2006; McPhate et al. 2000). Additionally, a pulse height filtering was applied to the FUV data to remove low and high gain events that are not related to actual photons. For the data used in this paper, only the very low and very high pulse height events were removed, keeping all the photons with a pulse height between 4 and 30 (the full range is between 0 and 31). For these observations, less than $0.7 \%$ of the data was filtered out. For both the FUV and NUV data, a dead-time correction was also applied to compensate for the loss of counts as the count rate increases. Most the the count rates were in the order of 50 counts/second for a deadtime correction of around $0.04 \%$. In the case of the NUV

\footnotetext{
${ }^{4}$ www.stsci.edu/hst/cos/documents/handbooks/current/toc.html
}

data, a flat field correction was also applied. There is currently no flat field for the FUV detector. The default extraction boxes for the spectra and the background areas were used with a bin size of $60 \mathrm{~s}$, a compromise to get a good enough $\mathrm{S} / \mathrm{N}$ on each data point and, at the same time, to sample adequately the pulsation cycles. Because of the much higher background noise seen with the NUV detector, smaller extraction regions for the spectra were chosen in this case in an attempt to minimize the noise contribution in the resulting count rates. Also, the regions where the coronal lines appear were removed from the extracted regions.

It turned out that all of the NUV light curves that we extracted were too noisy to be useful, except for those associated with our brightest target, SDSS J2200-0741 at $\mathrm{g}=17.70$. Indeed, we could not see obvious luminosity variations in the NUV light curves following a cursory look, and this was confirmed quantitatively by a Fourier analysis that failed to reveal periodicities with significant amplitudes above the (high) noise level in the light curves of each of the three pulsators known a priori. In contrast, and despite the fact that the count rates were lower in the FUV bandpass than in the NUV range, all of our FUV light curves proved themselves useful. In particular, obvious brightness variations are seen in the FUV light curves of the known pulsators.

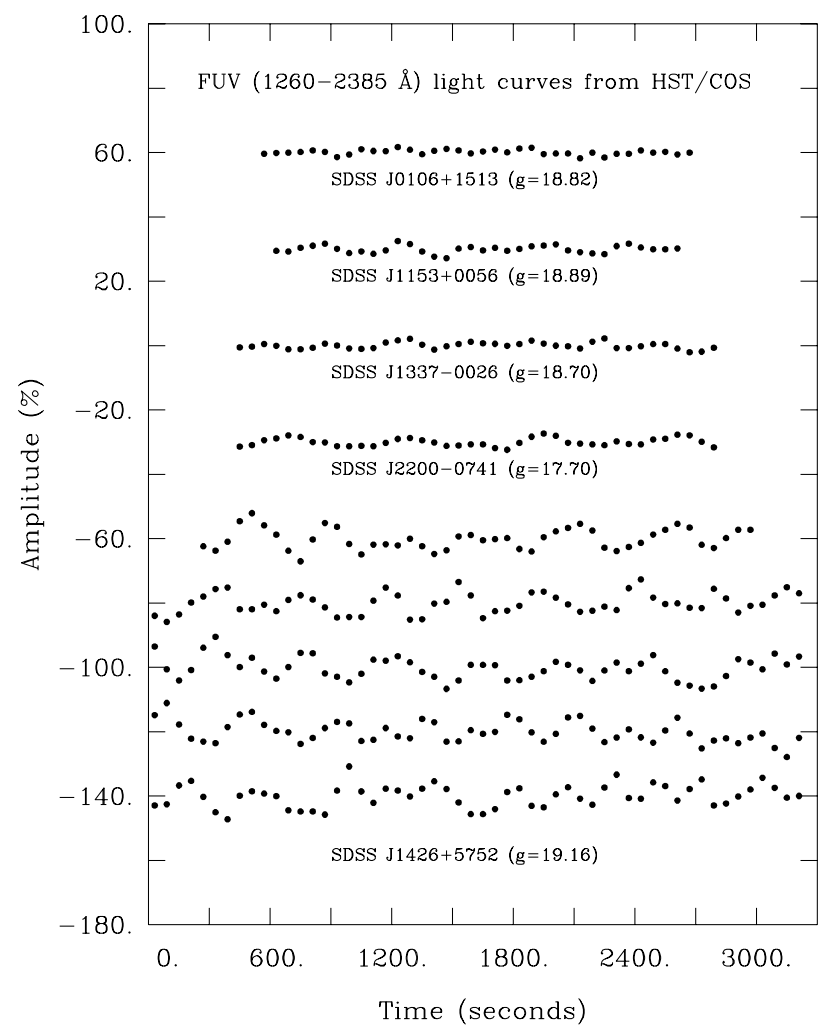

FIG. 1.- HST/COS light curves for the five observed Hot DQ white dwarfs from FUV G140L data (60 s bins).

In the end, we retained the light curves whose characteristics are summarized in Table 1. For each target, there is a FUV light curve segment available (five segments in the case of the faint object SDSS J1426+5752), 
TABLE 1

JOURNAL OF OBSERVATIONS

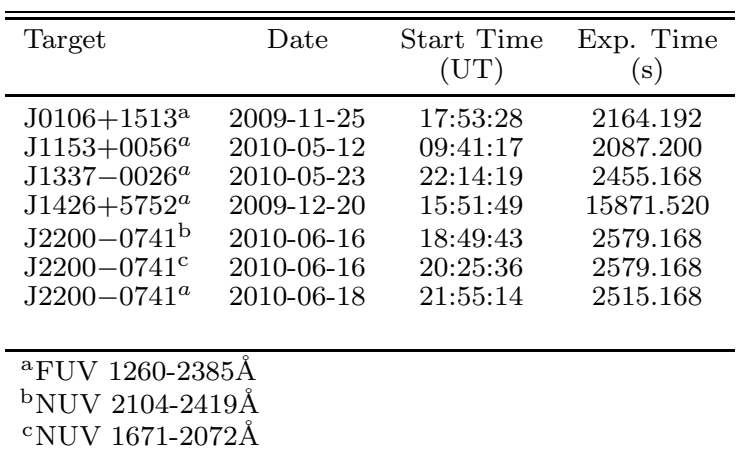

and, in addition, there are two NUV light curve segments available for SDSS J2200-0741. Figure 1 compares the FUV light curves for the five target stars. Luminosity variations are very clearly present in the three known pulsators, SDSS J1337-0026, SDSS J1426+5752, and SDSS J2200-0741, as well as in SDSS J1153+0056, a star that was not known to vary before. In contrast, photometric activity seems to be at a minimum in SDSS J0106+1513, another star whose variability status was unknown prior to this study.

\section{DISCUSSION}

We find it quite remarkable, especially in view of the relative faintness of our targets, that the HST/COS combination could pick up clear luminosity variations such as those illustrated in Figure 1. In order to verify the validity of our light curve extraction procedure, we first sought to recover published pulsation modes for each of the three known variable Hot DQ stars in our sample.

Because of its faintness, SDSS J1426+5752 has the best temporal coverage (15872 s) of all targets. We show the Fourier Power Spectral Density of the entire FUV data set (5 light curve segments) in the upper part of Figure 2. Clearly, the light curve is dominated by a single oscillation, and there is also a contribution from its first harmonic. Using standard procedures, we extracted two oscillations from the light curve, and the characteristics of these oscillations are summarized in the middle of Table 2 . We thus find a dominant pulsation mode with a period of $417.75 \pm 0.40 \mathrm{~s}$ and an amplitude of $3.16 \pm 0.33 \%$ of the mean brightness of the star in the FUV bandpass, a result that formally corresponds to a 7.5- $\sigma$ detection. The period corresponds remarkably well to the result of Green et al. (2009) who found a dominant periodicity of $417.7069 \pm 0.0008 \mathrm{~s}$ in the optical light curve of SDSS J1426+5752. The FUV amplitude of that pulsation mode is much larger than its counterpart in the optical domain (see Table 2), but this is expected from theoretical considerations (see below) and actually reinforces our findings.

Although the second oscillation picked up in our FUV light curve of SDSS J1426+5752 is formally only a $2.9-\sigma$ detection, it must be considered as "real" given that its period of $209.02 \pm 0.27 \mathrm{~s}$ is perfectly consistent with the value of $208.8534 \pm 0.0007 \mathrm{~s}$ found by Green et al. (2009)

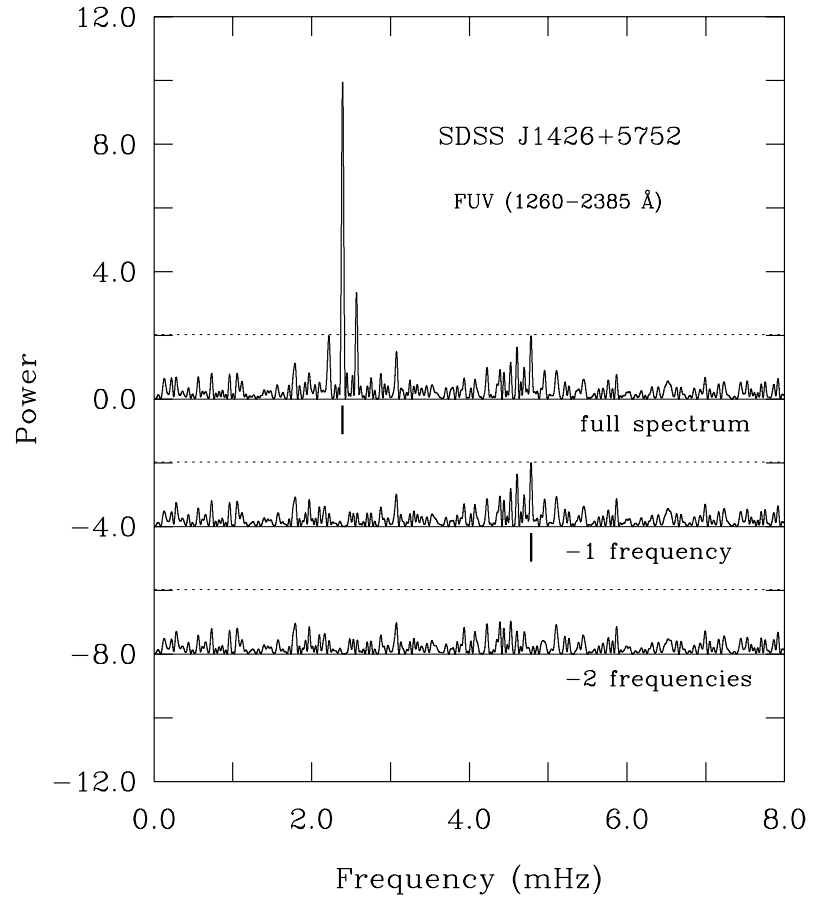

FIG. 2.- Fourier Power Spectral Density of the entire FUV data set (5 orbits) for SDSS J1426+5752 in the $0-8 \mathrm{mHz}$ range (upper curve). The lower transforms show the successive steps of prewhitening by the strongest frequency, and finally by the two frequencies that we isolated. The dotted horizontal lines show the 3- $\sigma$ detection level.

in the optical bandpass. In Green et al. (2009), as is the case here, that periodicity corresponds to the second highest peak in the Fourier transform of the light curve of the star, and is associated with the first harmonic of the dominant mode. Again, the FUV amplitude of that oscillation is significantly larger than the value of the amplitude in the optical range.

In the case of SDSS J2200-0741, we combined the available FUV light curve with the two NUV light curves to obtain a total coverage of $7673 \mathrm{~s}$. The results of our analysis are presented at the bottom of Table 2 . We thus found two significant periodicities $(653.17 \pm 0.16 \mathrm{~s}$ and $326.90 \pm 0.04 \mathrm{~s}$ ) which correspond again remarkably well to the two dominant oscillations $(654.397 \pm 0.056 \mathrm{~s}$ and $327.218 \pm 0.017 \mathrm{~s})$ observed in the optical light curve of that star by Dufour et al. (2009). Specifically, in both the COS spectral range and in the optical, the light curve of SDSS J2200-0741 is dominated by an oscillation and its first harmonic, the latter having an amplitude only slightly less than its parent mode. And the amplitudes of both periodicities are higher in the ultraviolet range than in the optical region.

Given that the other targets, including the third known pulsator SDSS J1337-0026, have only a short FUV light curve available each for analysis, we also reported the results of another frequency extraction exercise from a single FUV light curve for SDSS J1426+5752 in Table 2, and similarly for SDSS J2200-0746. Even though the uncertainties on the derived periods and amplitudes are substantially higher on the basis of these shorter data sets, Table 2 indicates clearly that the known pulsation modes are again well recovered.

Figure 3 is a montage of the Fourier transforms of five 
TABLE 2

Periodicities Detected in the HSt/COS Light Curves of Hot DQ White Dwarfs

\begin{tabular}{|c|c|c|c|c|c|c|c|}
\hline Star & $\begin{array}{l}\text { Period } \\
(\mathrm{s})\end{array}$ & $\underset{(\%)}{\text { Amplitude }}$ & $\mathrm{S} / \mathrm{N}$ & $\begin{array}{l}\text { Optical Period } \\
(\mathrm{s})\end{array}$ & Optical Amplitude & $\mathrm{S} / \mathrm{N}$ & Reference \\
\hline $\mathrm{J} 1153+0056$ & $\begin{array}{l}374.4 \pm 6.2 \\
159.1 \pm 1.5\end{array}$ & $\begin{array}{l}1.39 \pm 0.23 \\
1.06 \pm 0.23\end{array}$ & $\begin{array}{l}4.8 \\
3.7\end{array}$ & & & $\begin{array}{l}\cdots \\
\cdots\end{array}$ & this work \\
\hline J1337-0026 & $326.6 \pm 5.3$ & $1.14 \pm 0.25$ & 3.6 & $331-341$ & $0.23-0.40$ & 4.7 & Dunlap et al. (2010) \\
\hline $\mathrm{J} 1426+5752^{\mathrm{a}}$ & $\begin{array}{l}410.4 \pm 6.1 \\
210.5 \pm 2.2\end{array}$ & $\begin{array}{l}2.99 \pm 0.64 \\
2.16 \pm 0.64\end{array}$ & $\begin{array}{l}4.4 \\
3.2\end{array}$ & $\begin{array}{l}417.7069 \pm 0.0008 \\
208.8534 \pm 0.0007\end{array}$ & $\begin{array}{l}1.630 \pm 0.048 \\
0.487 \pm 0.048\end{array}$ & $\begin{array}{l}27.2 \\
8.1\end{array}$ & Green et al. (2009) \\
\hline $\mathrm{J} 1426+5752^{\mathrm{b}}$ & $\begin{array}{l}417.75 \pm 0.40 \\
209.02 \pm 0.27\end{array}$ & $\begin{array}{l}3.16 \pm 0.33 \\
1.20 \pm 0.33\end{array}$ & $\begin{array}{l}7.5 \\
2.9\end{array}$ & $\begin{array}{l}417.7069 \pm 0.0008 \\
208.8534 \pm 0.0007\end{array}$ & $\begin{array}{l}1.630 \pm 0.048 \\
0.487 \pm 0.048\end{array}$ & $\begin{array}{c}27.2 \\
8.1\end{array}$ & Green et al. (2009) \\
\hline $\mathrm{J} 2200-0741^{\mathrm{c}}$ & $\begin{array}{c}628.0 \pm 12.2 \\
330.5 \pm 5.5\end{array}$ & $\begin{array}{l}1.40 \pm 0.21 \\
0.86 \pm 0.21\end{array}$ & $\begin{array}{l}5.7 \\
3.5\end{array}$ & $\begin{array}{l}654.397 \pm 0.056 \\
327.218 \pm 0.017\end{array}$ & $\begin{array}{l}0.800 \pm 0.036 \\
0.655 \pm 0.036\end{array}$ & $\begin{array}{l}17.8 \\
14.6\end{array}$ & Dufour et al. (2009) \\
\hline $\mathrm{J} 2200-0741^{\mathrm{d}}$ & $\begin{array}{l}653.17 \pm 0.16 \\
326.90 \pm 0.04\end{array}$ & $\begin{array}{l}1.12 \pm 0.14 \\
1.02 \pm 0.14\end{array}$ & $\begin{array}{l}6.2 \\
5.7\end{array}$ & $\begin{array}{l}654.397 \pm 0.056 \\
327.218 \pm 0.017\end{array}$ & $\begin{array}{l}0.800 \pm 0.036 \\
0.655 \pm 0.036\end{array}$ & $\begin{array}{l}17.8 \\
14.6\end{array}$ & Dufour et al. (2009) \\
\hline
\end{tabular}

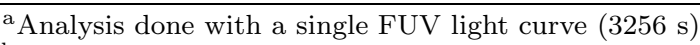

b Analysis done with the 5 FUV light curves (15872 s)

${ }^{\mathrm{c}}$ Analysis done with the single FUV light curve available (2515 s)

dAnalysis done with 3 light curves (FUV and NUV combined; $7673 \mathrm{~s}$ )

FUV light curves illustrated in Figure 1, one each for our five different targets. Note that the other small peak above $3 \sigma$ near $4 \mathrm{Mhz}$ for SDSS J1426+5752 in Figure 3 is a blend with the structure of the first harmonic. After prewhitening by the dominant mode and its first harmonic, no peaks above $3 \sigma$ remain. Similarly, no peaks above $3 \sigma$ are found in any of the 4 other light curves of SDSS J1426+5752. We can thus be highly confident that the short light curves are reliable and can be used to test for variability.

Related to that figure, Table 2 also includes the results of our analysis of the light curves of the three other stars in that sample beyond SDSS J1426+5752 and SDSS J2200-0741. In the case of the third known pulsating object, SDSS J1337-0026, we recovered easily (3.6- $\sigma$ detection) the main pulsation mode reported by Dunlap et al. (2010). However, it is interesting to note that the high amplitude harmonic periodicity that Dunlap et al. (2010) found at $\sim 169 \mathrm{~s}$ is absent in our dataset. Had an harmonically related periodicity as large as that reported by Dunlap et al. (2010) been present in our dataset, it would have been easily detected with our approach. The fact that the amplitudes of the detected modes in all three known pulsating targets are larger in the FUV than in the optical is a consequence of the wavelength dependence of the limb darkening law as explained in Fontaine \& Brassard (2008; see their Fig. 28 for instance). The amplitude-color relationship bears the signature of the degree index $\ell$ of a pulsation mode.

Figure 3 also reveals that SDSS J0106+1513 is not observed to vary (NOV), at least at a detectable level (limit of $1.18 \%$ in the $0-8 \mathrm{mHz}$ range), while SDSS J1153+0056 shows two distinct not harmonically related oscillations in its light curve, consistent with what is seen in Figure 1. The $374.4 \mathrm{~s}(159.2 \mathrm{~s})$ periodicity is a $4.8-\sigma(3.7-\sigma)$ detection and it has a $0.000063 \%(0.11 \%)$ chance of being due to noise according to the false alarm probability formalism proposed by Kepler (1993). We take this as the formal proof that these two oscillations correspond to real pulsation modes in SDSS J1153+0056, thus making it the first pulsating white dwarf to have been discovered from space-based observations. This underlines the potential of the time-tag capability of the HST/COS

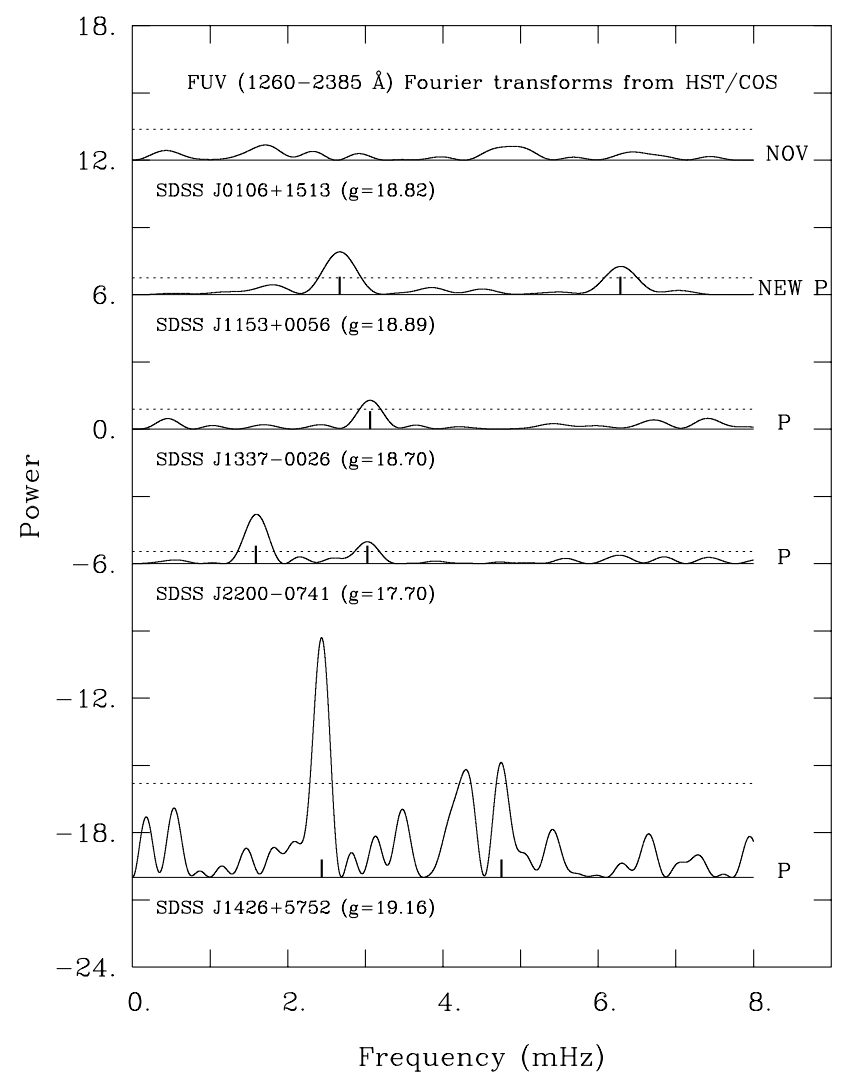

Fig. 3.- Fourier Power Spectral Density from 5 of the FUV light curves shown in Fig. 1 (only the top one is used in the case of SDSS J1426+5752). The dotted horizontal lines show the 3- $\sigma$ detection level ( 3 times the noise level in the bandpass of interest after prewhitening of the detected modes). Pulsation modes are recovered in the three known variable stars, while oscillations are revealed for the first time in SDSS J1153+0056

combination. Taking into account the rather short time bases involved here and the fact that four out of five targets showed detectable luminosity variations, pulsations could very well be a common characteristic of all Hot DQ white dwarfs when observed at high enough sensitivity.

This work was supported in part by the NSERC of Canada and FQRNT Québec. P.D is a CRAQ postdoc- 
toral fellow and P.B. is a Cottrell Scholar of Research for Science Advancement. G.F. acknowledges the contribution of the Canada Research Chair Program. This publication makes use of data from the HST proposal 11720 (PI: P. Dufour).

\section{REFERENCES}

Barlow, B. N., Dunlap, B. H., Rosen, R., \& Clemens, J. C. 2008, ApJ, 688, L95

Béland, S., \& Penton, S. V. 2006, Astronomical Data Analysis Software and Systems XV, 351, 339

Dufour, P., Liebert, J., Fontaine, G., \& Behara, N. 2007, Nature, 450, 522

Dufour, P., Fontaine, G., Liebert, J., Schmidt, G. D., \& Behara, N. 2008, ApJ, 683, 978

Dufour, P., et al. 2009, ApJ, 703, 240

Dufour, P., et al. 2010, 17th European Workshop on White Dwarfs, AIP Conf. Proc. 1273, 64
Dunlap, B. H., Barlow, B. N., \& Clemens, J. C. 2010, ApJ, 720, L159

Fontaine, G., \& Brassard, P. 2008, PASP, 120, 1043

Fontaine, G., Brassard, P., \& Dufour, P. 2008, A\&A, 483, L1

Green, E. M., Dufour, P., Fontaine, G., \& Brassard, P. 2009, ApJ, 702,1593

Kepler, S.O. 1993, Balt. Astron., 2, 515

McPhate, J. B., Siegmund, O. H., Gaines, G. A., Vallerga, J. V., \& Hull, J. S. 2000, Proc. SPIE, 4139, 25

Montgomery, M. H., Williams, K. A., Winget, D. E., Dufour, P., DeGennaro, S., \& Liebert, J. 2008, ApJ, 678, L51 